
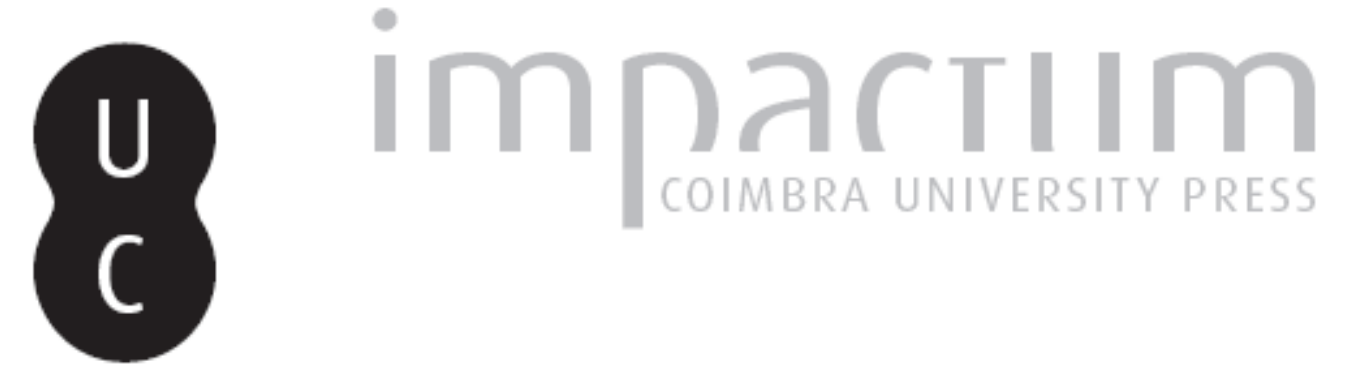

\title{
Tradizione, novità e fortuna in età moderna del De mulierum virtutibus di Plutarco
}

\section{Autor(es): Paci, Valentina}

Publicado por: International Plutarch Society

URL persistente:

URI:http://hdl.handle.net/10316.2/37640

DOI:

DOI:http://dx.doi.org/10.14195/0258-655X_5_5

Accessed : $\quad$ 26-Apr-2023 09:47:03

A navegação consulta e descarregamento dos títulos inseridos nas Bibliotecas Digitais UC Digitalis, UC Pombalina e UC Impactum, pressupõem a aceitação plena e sem reservas dos Termos e Condições de Uso destas Bibliotecas Digitais, disponíveis em https://digitalis.uc.pt/pt-pt/termos.

Conforme exposto nos referidos Termos e Condições de Uso, o descarregamento de títulos de acesso restrito requer uma licença válida de autorização devendo o utilizador aceder ao(s) documento(s) a partir de um endereço de IP da instituição detentora da supramencionada licença.

Ao utilizador é apenas permitido o descarregamento para uso pessoal, pelo que o emprego do(s) título(s) descarregado(s) para outro fim, designadamente comercial, carece de autorização do respetivo autor ou editor da obra.

Na medida em que todas as obras da UC Digitalis se encontram protegidas pelo Código do Direito de Autor e Direitos Conexos e demais legislação aplicável, toda a cópia, parcial ou total, deste documento, nos casos em que é legalmente admitida, deverá conter ou fazer-se acompanhar por este aviso.

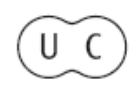




\title{
Tradizione, novità e fortuna in età moderna del De mulierum virtutibus di Plutarco di
}

\author{
Valentina Paci \\ Università di Bologna \\ v.paci@libero.it
}

Abstract

\begin{abstract}
Plutarch's idea of women is different from the one of the Greek misogynist writers. In De mulierum virtutibus Plutarch collected twenty-seven exemples of women

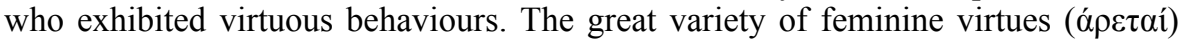
which Plutarch included in his work can explain the great fortune of the essay in Modem Age. The essay could be interpreted in a conservative or revolutionary way, therefore it could be used either to defend women or to denigrate them. In this paper I deal with also an edition of Plutarch's De mulierum virtutibus (published in Piacenza in 1794) on which it has never been done a research up to now. It is an abridged version of the original essay written by Plutarch containing only twelve exemples of virtuous behaviours.
\end{abstract}

Key-Words: Plutarch, De mulierum virtutibus, Ancient Women, Feminine Virtues, Literary Tradition of Plutarch, European Humanism.

$\S 1$.Il rapporto del De mulierum virtutibus di Plutarco con la tradizione greca misogina precedente

Nell'incipit del De mulierum virtutibus Plutarco polemizza apertamente con Tucidide, che in un famoso passo del libro II delle Storie afferma che la donna migliore è quella della quale si parlerà meno, nel bene o nel male ${ }^{1}$. Che la donna non dovesse far parlare di sé, né potesse parlare in pubblico è ben noto ${ }^{2}$. Recentemente P. Burian in uno studio dedicato alle Troiane di Euripide, ha sostenuto che alle donne era concesso prendere la parola solo nello spazio privilegiato del teatro; pertanto il fatto che il loro discorso fosse "proibito altrove nella polis rende l'eccezione ancora più

1 Thuc., II 45. 2. Sulla polemica di Plutarco contro lo storico greco cf., in particolare, M. GARCİA VALDÉS, "Plutarco versus Tucidides: Virtutes Mulierum", in M. JUFRESA, F. Mestre, P. Gómez \& P. Gilabert (eds.), Plutarc a la seva època: paideia i societat. Actas del Vili Simposio Espanol sobre Plutarco (Barcelona, 6-8 de Novembre de 2003), Barcelona, 2005, pp. 297-312.

2 Sull'esclusione delle donne dalla vita politica nel mondo greco e romano si vedano N. LorauX, "La cité, l'historien, les femmes", Pallas, 32 (1985) 7-29 e C. Petrocelli, La stola e il silenzio. Sulla condizione femminile nel mondo romano, Palermo, 1989. 
evidente e quindi più efficace"3.

La tradizione misogina nella quale Tucidide si inserisce aveva comunque origini ben più antiche: per comprenderlo è sufficiente menzionare i versi della Teogonia 4 di Esiodo in cui la "razza" delle donne è ritenuta l'origine di tutti i mali per l'umanità o ricordare il famoso Giambo contro le donne di Semonide di Amorgo, incentrato sul paragone fra donne ed animali. Tale filone misogino troverà nel IV secolo a.C. tra i suoi principali esponenti Aristotele - convinto assertore dell'inferiorità per natura ( $\varphi v$ $\sigma \alpha)$ della donna ${ }^{5}$ - le cui teorie influenzeranno pesantemente il pensiero occidentale fino al Seicento ${ }^{6}$.

Dunque, al pregiudizio di Tucidide contro le donne, già da lungo tempo for- temente radicato nella tradizione greca, il Cheronese contrappone la bontà del costume romano (o 'P $\omega \mu \alpha i ́ \omega v$ vó $\mu$ os) di

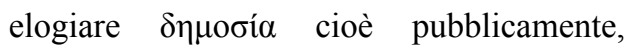
le donne nella laudatio funebris. La convinzione che queste, come gli uomini, possano dare prova di gesta virtuose ed eroiche induce Plutarco a dedicare attenzione anche a personaggi femminili illustri ${ }^{7}$ : egli ha ritenuto le donne degne di

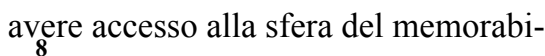
$\mathrm{le}^{\mathbf{8}}$. La diversa opinione che Plutarco ha delle donne è del resto già testimoniata dal fatto che l'opera è dedicata ad una sacerdotessa, Clea, colei alla quale è rivolto anche il De Iside et Osiride ${ }^{9}$.

Nel proemio l'autore dichiara di voler dimostrare attraverso il ricorso ad exempla concreti ${ }^{10}$ - collettivi ed indivi-

3 P. Burian, "Voce di donna: Le Troiane nella guerra del Peloponneso", in A. CASANOva e P. DESIDERI (eds.), Evento, racconto, scrittura nellantichità classica. Atti del convegno internazionale di studi Firenze, 25-26 novembre 2002, Firenze, 2003, pp. 35-53.

${ }^{4}$ Hes., Th.592,600-601.

5 Arist., Poi. 1252 a-b; $125 \%$ e 1260 a.

6 Lucrezia Marinelli cercherà di "distruggere" le teorie misogine aristoteliche. Cf. infra. p. 78 e nota 52 .

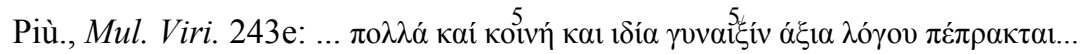

8 G. POMATA, 1990, p. 348.

Clea, che condivideva con Plutarco il gusto per la filosofia, aveva affidato al Cheronese sua figlia, Memmia Euridice, perché egli la educasse. Cf., per altre notizie sulla donna B. Puech, "Prosopographie des amis de Plutarque", Aufstieg und Niedergang der Rõmischen Welt, 2. 33. 6, Berlin, New York, 1992, 4842-4843. Testo di riferimento imprescindibile per lo studio del De mulierum virtutibus di Plutarco resta P. A. STADTER, Plutarch s historical methods. An Analysis of the Mulierum Virtutes, Cambridge, 1965.

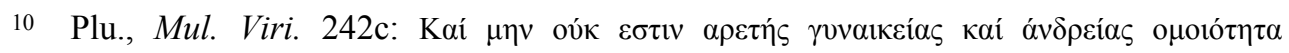

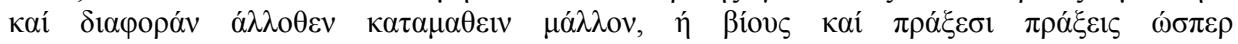

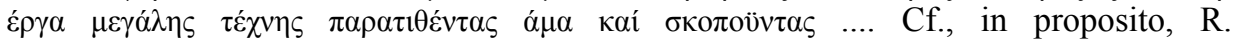
R. J. BenefIEL, "Teaching by example. Aetiology in Plutarch's De Mulierum Virtutibus", Ploutarchos, n.s., 1 (2003/2004) 11-20. 
duali - di virtù e coraggio testimoniati da donne di ogni tempo e appartenenti alle più svariate realtà geografiche ${ }^{11}$, che "la virtù degli uomini e delle donne è

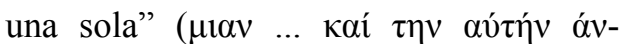

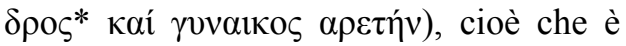
identica ed universale ${ }^{12}$. Il Cheronese è indubbiamente portatore di una nuova concezione riguardo al genere femminile: lo scrittore, senza distogliere la donna dalla sua missione naturale, senza cancellare i limiti a lei imposti dalla natura, senza equipararla del tutto all'uomo, la cui posizione egli intende salvaguardare, sostiene l'idea che essa, ricevendo un'educazione simile a quella dell'uomo, possa essere elevata ad un grado spirituale pari a quello maschile ${ }^{13}$; Plutarco del resto era convinto della insegnabilità della virtù - tema al quale aveva dedicato il trattato intitolato $A n$ virtus doceri possit - e della necessità di educare anche le donne, come testimonia lo scritto perduto a lui attribuito da

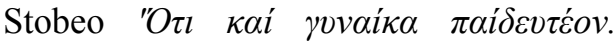
Non solo. Plutarco riteneva le donne degne di essere istruite negli studi più elevati, quali la geometria - utile a distogliere la donna da pratiche sconvenienti come la danza e ad allontanarla da superstizioni e false credenze $^{14}$ - e la filosofia. Il rispetto e la diversa considerazione che il Cheronese mostra di avere nei confronti delle donne molto risente sia dell'influsso del pensiero stoico

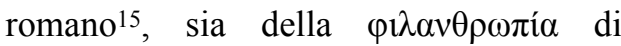
Plutarco, una virtù che si avvicina al principio cristiano di benevolenza e di amore verso il prossimo, nonostante tale principio non abbia, di per sé, alcuna relazione diretta con il Cristianesimo. La posizione morale che fa apparire Plutarco vicino al Cristianesimo e che determinerà la fortuna di molti suoi

11

Questo modo di procedere sembra giustificato dalla volontà di dimostrare che la virtù è caratteristica che appartiene universalmente alle donne di ogni tempo. Piu., Mul. Viri. 242f.

13 K. Ziegler, Plutarco, p. 367 e, più recentemente, J. Boulogne, 2005, pp. 225-233. La donna ben educata ed istruita è in grado di condividere appieno con il proprio marito anche la vita intellettuale: cf. R. HIRZEL, 1912, p. 29.

14 Piu., Praec. con. $148 \mathrm{c}$.

15 Sotto l'influsso dello Stoicismo erano nate opere quali quella di Cleante (La virtù è identica nelluomo e nella donna), di Musonio Rufo, contemporaneo di Vespasiano che scrive due opere dal titolo Se si debbano educare le figlie nello stesso modo dei figli (Et

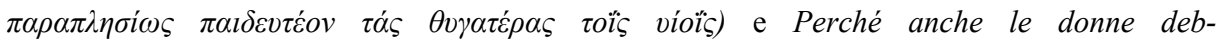

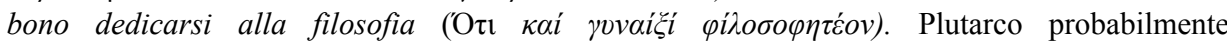
venne influenzato da questa produzione letteraria, come sostengono Y. VERNIÈRE, "Plutarque et les femmes", AncW, 25 (1994), p. 167 e J. Boulogne, 2005, p. 233. Cf. anche D. BABUt, Plutarco e lo Stoicismo, ed. italiana a cura di A. RADICE, Milano, 2003, pp. 127 ss. 
scritti presso i padri della Chiesa ${ }^{16}$ è, in realtà, come ha già sostenuto Ziegler, il frutto degli insegnamenti che venivano da Socrate, da Platone, dall'Accademia, dai Peripatetici e, appunto, dalla Stoa ${ }^{17}$.

Plutarco non è certo il primo a comporre un catalogo delle donne, genere antichissimo, le cui origini risalgono già ad Omero ed Esiodo ${ }^{18}$, ma il De mulierum virtutibus pur riagganciandosi a tale genere, presenta - per volontà stessa del suo autore - un'impostazione assolutamente originale ed innovativa. Plutarco, infatti, prendendo le distanze dalla lunga tradizione precedente e scardinando canoni già consolidati, sceglie deliberatamente di dare voce a personaggi femminili di cui nessuno aveva mai parlato prima $^{19}$ - ciò vale per diciotto storie delle ventisette complessive - e di riferire dettagli omessi dagli altri scrittori per le restanti nove ${ }^{20}$. I cataloghi delle donne illustri cui Plutarco allude dovevano essere numerosi. Purtroppo, tali opere sono andate quasi tutte perdute e la tradizione per lo più ne conserva solo i titoli. Carone di Cartagine, secondo il lessico Suda, avrebbe composto un'opera dedicata a uomini e donne illustri inti-

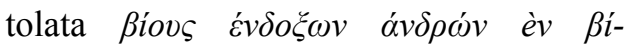

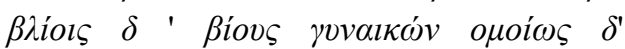
Un certo Sopatro avrebbe realizzato excerpta dell'opera composta dallo stoico Apollonio - vissuto nel primo secolo

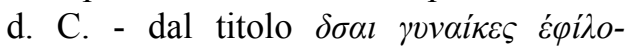

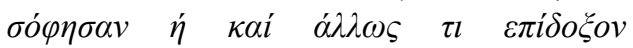

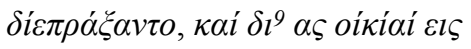

M. La Matina, Plutarco negli autori cristiani greci, in I. GALlo (ed.), L 'eredità culturale di Plutarco dall'antichità al Rinascimento. Atti del VII Convegno plutarcheo Milano-Gargnano, 28-30 maggio 1997, Napoli, 1998, pp. 81-110.

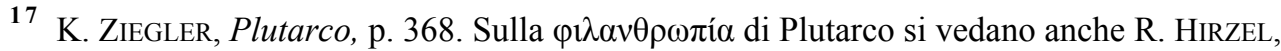
1912, p. 24 ss. e, più recentemente, H. MARTIN, "The Concept of philanthropia in Plutarch's Lives", AJPh, 82 (1961) 164-175; L. VAN JOHNSON, "The Humanism of Plutarch", CJ, 66 (1970) 26-37; S-T. TEODORSSON, "El programa de Plutarco para la conducta social" in M. Jufresa, F. Mestre, P. Gómez, P. Gilabert (eds.), Plutarc a la seva època: paideia i societat, Barcelona, 2005, pp. 659-664.

18 Cf. Horn., Od. 11.225-247, dove sono enumerate le regine e le principesse che circondano Ulisse. Per quanto riguarda Esiodo, al poeta sono attribuiti II catalogo delle donne e Le grandi Eoie. Sulla natura di questi cataloghi delle donne cf. M. L. WeST, The Hesiodic Catalogue of Women. Its Nature, Structure, and Origins, Oxford, 1985 e le osservazioni di D. GERA, 1997, pp. 40-44. Tali cataloghi si presentavano per lo più come dei poemi genealogici o come compendi di miti eroici.

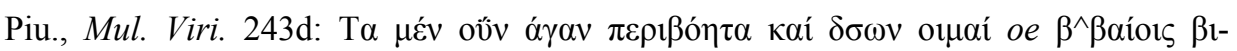

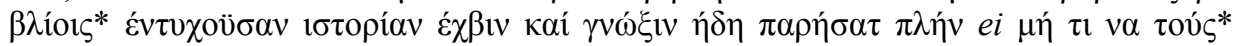

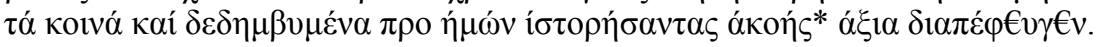

20 Gli episodi in questione riguardano Troiane, Focesi, Argive, Persiane, Tirrene, Valeria e Clelia, Pieira, Policrita, Chiomara. 
evvotav $\sigma v v \varepsilon ́ \pi \rho \alpha \chi \theta \eta \sigma \alpha v^{21}$. Inoltre D. Gera, che ha analizzato con attenzione il Tractatus de mulieribus claris in bello, opera - secondo la studiosa - da attribuirsi ad un'autrice anonima e da datarsi approssimativamente ad un periodo tra il II e il I secolo a. C., potrebbe avere costituito una possibile fonte di Plutarco $^{23}$. Sta comunque di fatto che, forse per le particolari caratteristiche che presenta, $\mathrm{o}$, molto più probabilmente, per l'enorme fortuna conosciuta dal suo

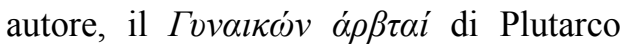
è sfuggito al naufragio che hanno invece subito molti altri trattati appartenenti a questo genere. Sarà infatti questo opuscolo a costituire un punto di riferimento imprescindibile a partire dalla prima età moderna, quando $\mathrm{i}$ cataloghi delle donne illustri cominceranno ad essere consapevolmente utilizzati all'interno di vivaci dibattiti letterari sulla condizione e la natura femminile.

Molti studiosi tendono soprattutto in tempi recenti a sminuire notevolmente la portata innovativa del De mulierum virtutibus sostenendo che le azioni virtuose di cui le eroine elogiate dal Cheronese danno prova andrebbero riferite a situazioni eccezionali di grave pericolo, nelle quali spesso è la libertà della loro comunità di appartenenza ad essere minacciata dalla tirannide ${ }^{24}$. Solo condizioni estreme giustificherebbero pertanto l'assunzione, da parte delle donne, di ruoli maschili che in altre circostanze sarebbe stata loro perentoriamente interdetta. La vivacità del dibattito attuale inteso a stabilire quale idea Plutarco avesse delle donne $^{25}$ e le molteplici interpretazioni che gli studiosi danno sia dell'opuscolo

21 Per ulteriori dettagli D. GERA, 1997, pp. 33-35.

22

Più propriamente, il titolo che è dato all'opera nei manoscritti è $\Gamma v v \alpha i ́ \kappa \varepsilon \varsigma^{*}$ ev $\pi \mathrm{o} \lambda \varepsilon \mu \mathrm{t}-$

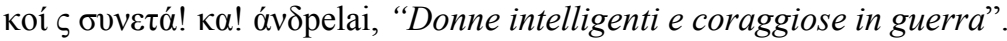

23 D. GERA, 1997, p. 35.

24 K. O'BRIEN WICKER, 1978, p. 120: “...it should be noted that most of his exemples in the Mulierum virtutes focus on the behavior of women in extraordinary situations, most often when the freedom of community in being threatened by tyranny"; più recentemente M. H. DetTenhofer, "Frauenbilder in Plutarchs Schrift Mulierum Virtutes im Verhältnis zum traditionellen Frauenbilder der Griechen", in C. ULF, R. ROLLINGER (eds.), Geschlechter - Frauen - Fremde Ethnien. In antiker Ethnographie, Theorie und Realität, Innsbruck, Wien, München, Bozen, 2002, p. 433: “...es geht... um Frauen, die sich in Ausnahmesituationen in traditionellen Männerdomänen bewährten. [...] Sie finden in Ausnahmesituationen, häufig extremer militärischer Bedrohung oder drückender Tyrannenherrschaft statt, und sie erfolgen zum Wohle der Polis und/oder aus bedingungsloser Loyalität gegenüber den Ehemännern”. to Aretaphila. Women in Politics in Plutarch", in J. Mossman (ed.), Plutarch and his Intellectual World, London, 1997, pp. 73-74. 
sia del concetto di virtù femminile che vi è sotteso, dimostra che l'opera continua oggi ad essere letta e interpretata in modi antitetici e contrapposti, così come, in fondo, è avvenuto anche in passato. Come si vedrà in seguito, paradossalmente il De mulierum virtutibus in età moderna è stato utilizzato ora dalle femministe per difendere l'onore e la dignità del sesso femminile, ora dai "conservatori" per richiamare la donna al ruolo tradizionale di moglie, madre, educatrice. Come ha sostenuto K. O'Brien Wicker ${ }^{26}$, il trattato di Plutarco nel suo complesso offriva non un solo modello di donna virtuosa, ma molteplici esempi di donne valorose, che danno prova di differenti $\alpha \rho^{\wedge} \tau \alpha i$. Il Cheronese elogia così le Troiane per il loro $\lambda \mathrm{o} \gamma 1 \sigma \mu \mathrm{ó}_{\varsigma^{27}}$, le Chiote per $\tau$ o $\lambda \mu \alpha$ e $\theta \alpha \rho \rho \beta_{i v}^{28}$, le Argive per o $\mu \mu$ e $\tau$ ó $\lambda \mu \alpha^{29}$, Erixo per $\sigma \omega \varphi \rho о-$

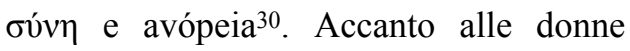
esaltate per il coraggio e l'ardimento dimostrato in situazioni di pericolo, si pongono personaggi femminili che si distinguono per comportamenti virtuosi nel privato, come Camma ${ }^{31}$, lodata per l'amore nutrito in vita nei confronti del proprio marito e per la $\tau \tau i ́ \sigma \tau ı \varsigma$ mantenuta anche dopo la morte del consorte.

Plutarco resta comunque un innovatore anche solo per il fatto di avere lasciato che nel suo opuscolo fosse la donna aggressiva, guerriera, coraggiosa ed ardimentosa a campeggiare, come dimostra il fatto che quantitativamente sono più numerosi gli esempi di figure femminili che presentano caratteristiche ritenute tradizionalmente maschili. Ciò non significa che Plutarco fosse convinto che le donne dovessero realmente fare guerre o governare stati e città: grande e impareggiabile è la virtù di Aretafila, che per avere ucciso il tiranno di Cirene ed avere restituito la libertà ai cittadini, riceve la proposta di prendere parte attiva alla vita politica della città insieme agli uomini migliori ${ }^{32}$. Ancora più grande ed elogiabile doveva apparire, agli occhi di

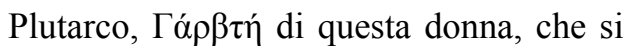
dimostrò capace di rifiutare al momento opportuno tale richiesta, scegliendo di ritirarsi a vita privata.

K. O'BRIEN WICKER, 1978, p. 109: "Taken as a whole, the stories constitute a composite picture of the ideal woman: virtuous in private moral behavior; concerned with the good of the community; able to contribute intelligently, justly and piously to its welfare; courageos and resourcefulk in defending it against enemies; and willing to die and to see her husband and children killed rather than to endure tyranny".

Plu., Mul. Virt. 243e.

28 Plu., Mul. Virt. 245a.

29 Plu., Mul. Virt. 245d.

30 Plu., Mul. Virt. 26Id.

31 Plu., Mul. Virt. 258b-c; 258e-f.

32 Plu., Mul. Virt. 257d. 
§2. La fortuna del De mulierum virtutibus di Plutarco in età moderna e il catalogo delle donne illustri

L'ampio recupero del De mulierum virtutibus di Plutarco nella modernità è stato indubbiamente favorito dal fatto che l'opera era ben nota agli umanisti ${ }^{33}$, dai quali venne particolarmente apprezzata. Il numero di edizioni che vengono prodotte soprattutto a partire dalla fine del Quattrocento testimonia in modo inequivocabile la particolare diffusione dell'opuscolo e l'interesse che esso suscitò. Riporto di seguito un elenco, ovviamente selettivo, di alcune tra le edizioni più note e significative dell'opera ${ }^{34}$ :

- edizione latina di Alamanus Ranutinus (1485);

- edizione fiorentina di Antonio Ridolfi che traduce l'opera dal latino e la dedica alla sua compatriota Maria Albizi de' Dei (1542);

- De virtutibus mulierum. Plutarco, Trattato delle virtù delle Donne, tradotto dal Greco in Latino da Alamanno Rinuccini ed in Italiano da Anonimo (non pervenutaci, ma della cui esistenza siamo informati grazie alla $\mathrm{Bi}$ blioteca degli volgarizzatori di Filippo Argelati del 1767);

edizione francese di Jean des Monstiers (1538), che dedica l'opera a Margherita di Francia (1523-1574);

- edizione di J. Amyot, Les vertueux faicts des femmes (1572), che venne tradotta in inglese da T. North nel 1579, cui seguirono anche edizioni successive (1595 e 1603).

Il De mulierum virtutibus rappresenta il trattato di Plutarco più frequentemente tradotto nel Cinquecento, assieme al De amico et adulatore e al De liberis educan$d i s^{35}$. I "satelliti" inglesi dell'edizione francese di Amyot sono a loro volta all'origine della fortuna e della diffusione dell'opuscolo nell'Inghilterra del ' $600^{36}$.

Alle notizie sopra riportate, va aggiunto che il testo del De mulierum virtutibus di Plutarco venne ritrascritto dal latino al greco dall'umanista C. Lascaris attorno al $1490^{37}$. A. Guzmàn Guerra, che si è occupato della questio-

33 In realtà, altre opere di Plutarco erano probabilmente già note nella seconda metà del Trecento: il De cohibenda ira infatti venne tradotto in latino ad Avignone nel 1372-3 e un testo aragonese delle Vite parallele venne preparato qualche anno più tardi. Cf., in proposito, R. WeISS, “Lo studio di Plutarco nel Trecento", PP, 8 (1953) 321-342.

34 Per notizie più dettagliate cf. R. Aulotte, Amyot et Plutarque. La tradition des Moralia au XVIe siècle, Genève, 1965, pp. 69 ss.

R. Aulotte, 1989, pp. 99-104.

36

D. A. RusSell, 1973, p. 152.

37 Il trattato occupa i fogli dal 24 al 34 del manoscritto 4621, conservato alla Biblioteca Nazionale di Madrid. 
ne, ha sottolineato la particolarità e, per certi versi, la stranezza di questo singolare testo delle Virtù delle donne, che pur non costituendo una testimonianza fondamentale per la trasmissione dell'opuscolo di Plutarco, è tuttavia la dimostrazione dell'amore profondo che il Lascaris come altri umanisti - nutriva per l'opera di Plutarco e del suo zelo bibliofilo, che lo indusse a ritradurre il trattato nella lingua in cui era stato scritto in origine. ${ }^{39}$

Dunque, la grande fortuna di questo particolare opuscolo nasce in età umanistica ${ }^{40}$, quando cominciano ad apparire numerose edizioni e traduzioni dell'opera. Esso si è prestato agli usi e alle forme di recupero più disparate in età moderna. Del resto, i cataloghi delle donne illustri costituivano, già per loro stessa natura, dei repertori di esempi concreti di virtù, funzionali non solo a fornire degli ammaestramenti, ma anche a difendere o, viceversa, ad attaccare il sesso fem- minile $^{41}$. Per questo l'opera è stata spesso richiamata nell'ambito del dibattito sul carattere e la natura della donna che prese avvio sul finire del XIV secolo e che sarebbe durato per tutta la prima età moderna. Il De Claris mulieribus di Boccaccio costituì la prima opera di questo genere dopo il De mulierum virtutibus di Plutarco a cui si ispirarono moltissimi autori di varia nazionalità nel corso dei successivi trecento anni, i quali inclusero nei loro trattati anche eroine bibliche, sante cristiane e figure a loro contemporanee. A prima vista il testo di Boccaccio e gli altri che seguirono potrebbero apparire un omaggio senza riserve al sesso femminile, ma a torto ${ }^{42}$. Boccaccio ritiene infatti che la natura abbia dato alle donne un corpo debole $\boldsymbol{e}$ un ingegno tardo $^{43}$. A questa teoria misogina Christine de Pizan - la prima donna che intervenne nel dibattito, da alcuni ritenuta la prima femmini-
38

39 Sulle mutilazioni che sono state compiute - mancano qui gli episodi relativi alle donne della Licia e alle Salmatidi - si può supporre, secondo A. GUZMÀN GUERRA, 1990, p. 268 che le omissioni non siano state volontarie, ma che dipendano dal fatto che tali episodi, probabilmente, non comparivano neppure nel manoscritto latino al quale il Lascaris avrebbe fatto riferimento.

R. Aulotte, 1989, pp. 100 ss. : "Que ce soit en Italie ou en France, Plutarque, favorise, au total, la naissance d'un «humanisme humanisé», qui prend en compte les réalités de la permanente nature humaine et les diversités variables des circonstances historiques ou géographiques”. Del tema si è occupato D. A. RUSSELL, 1973, p. 145.

S. Bucci, "La produzione letteraria dedicata alle donne illustri. Pubblico e autori nel clima polemico del dibattito sui diritti del sesso femminile", in F. TARICONE \& S. Bucci (eds.), La condizione della donna nel XVII e XVIII see., Roma, 1983, p. 140.

43 BOCCACCIO, De Claris mulieribus, proemio, 4. 
sta e l'iniziatrice della Querelle des fem$m_{e s^{44}}$ - replica così nella Cité des dames (1405): "Se qualcuno vuole sostenere che le donne non sono abbastanza intelligenti per imparare il diritto, la storia dimostra il contrario; come vedremo in seguito, molte donne sono state grandi filosofe e hanno dominato scienze ben più complesse e più nobili di quanto non siano le leggi scritte e le regole stabilite dagli uomini" ${ }^{45}$. Da questi accesi dibattiti sono scaturite inoltre letture e interpretazioni contrapposte di personaggi come Semiramide, che in Boccaccio ha tutte le caratteristiche della regina crudele e libidinosa ${ }^{46}$, mentre nella Cité des dames è una donna che dimostra grande virtù e coraggio nell'esercizio delle $\operatorname{armi}^{47}$. Analogamente, Didone descritta dall'autore del De Claris mulieribus come exemplum di fedeltà coniugale solo con il pretesto di sferrare l'attacco contro le vedove corrotte del suo tempo ${ }^{48}$, figura nell'opera di C. de Pizan tra le regine sagge: di lei sono esaltate soprattutto la prudenza e l'intelligenza ${ }^{49}$, nonché il ruolo di fondatrice della città di Cartagine. Nella prima età moderna, tuttavia, la Cité des dames ebbe poco credito. Nella De nobilitate et praecellentia foeminei sexus declamatio pubblicata ad Anversa nel 1529, l'umanista tedesco Cornelio Agrippa di Nettesheim fornì l'elenco di donne illustri più completo e più spesso imitato nel tempo, sostenendo la teoria della superiorità della donna sull'uomo. Non bisogna, tuttavia, lasciarsi ingannare dalle apparenze. L'aspetto più paradossale di questa storiografia risiede nella sua fondamentale ambiguità ed incertezza: "Si sceglie un criterio per parlare della donna, quello della sua celebrità, che è in fondo, la negazione delle più profonde convinzioni culturali della società e dello storico stesso"50. Del resto, già N. Zemon Davis, in un saggio di alcuni anni fa, riconosceva i limiti di questa storiografia e ribadiva che già il fatto stesso che esista un genere in cui si parla
44

Cf. J. Kelly, "Early Feminist Theory and the Querelle des Femmes, 1400-1789", Signs. Journal of Women in Culture and Society, 8 (1982) 4-28. Sul significato storico della Querelle des Femmes e le sue origini si veda anche, più recentemente, M. ZIMMERMANN, "La «Querelle des Femmes» come paradigma culturale", in S. SEIDEL MENCHI, A. JaCOBSON SChUtTe, T. KUEHN (eds.), Tempi e spazi di vita femminile tra medioevo ed età moderna, Bologna, 1999, pp. 157-173.

45

46

47

48

49

50

C. DE PIZAN, Cité des dames, cit., I, XI. Traduzione di P. CARAFFE

BOCCACCIO, De Claris mulieribus, II.

C. De PIZAN, Cité des dames, I, XV.

Boccaccio è anche autore del Corbaccio, feroce satira contro le donne.

Cf. il commento di P. CARAFFI, ed. cit., pp. 21-22.

G. CONTI Odorisio, 1979, p. 29. 
delle donne separatamente dagli uomini presuppone la loro marginalità ed esclusione dalla storia ${ }^{51}$. Ciò, tuttavia, non ha impedito che proprio le donne - sulla falsariga di Plutarco - siano ampiamente ricorse a trattati di questo genere per parlare in lode o in difesa del loro sesso. Lucrezia Marinelli, autrice de La nobiltà et l 'eccellenza delle donne co 'diffetti et mancamenti De gli Huomini. Discorso di Lucrezia Marinella in due parti diviso (Venezia 1601), utilizza exempla concreti di virtù femminile per distruggere la teoria misogina aristotelica ${ }^{52}$. La stessa Mdm. De Villedieu, pur scegliendo di scrivere non un catalogo delle donne illustri, ma degli Annales galantes de Grèce per rivendicare alle donne il ruolo di soggetto attivo della storia ${ }^{53}$, continua a tenere presente l'opera di Plutarco, non rinunciando ad utilizzare esempi concreti di virtù femminile per dare maggiore forza alle proprie argomentazioni ${ }^{54}$.

Dunque i cataloghi delle donne illustri si sono spesso prestati ad usi contrastanti. Anche nell'Ottocento molti autori hanno scelto di seguire l'esempio e il metodo di Plutarco per sostenere teorie del tutto antitetiche sui compiti e i ruoli da attribuire alle donne. Mettiamo a confronto due Plutarchi femminili, entrambi della seconda metà dell'Ottocento. Il primo, composto dal codino P. Fanfani ${ }^{55}$,

Cf. N. Z. DAvis, "Women's History" in Transition: The European Case, Feminist Studies, 3 (1976) 83-103.

Il discorso delTautrice si divide in due parti, come recita il sottotitolo dell'opera: "Nella prima si manifesta la nobiltà delle Donne co 'forti ragioni, \& infiniti essempi, \& non solo si distrugge Topinione del Boccaccio, d'amendue i Tassi, dello Sperone, di Monsig. di Namur, \& del Passi, ma d'Aristotile il grande anchor a. Nella seconda si conferma co vere ragioni, \& co 'varij essempi da innumerabili Historici antichi, e moderni tratti, Che i Diffetti de gli huomini trapassano di gran lunga que'delle Donne".

M. De VILledieu, Annales galantes de Grèce, Genève, 1971, pp. 369-370: “Cependant il me semble que les Nations étant composées de deux Sexes, on ne peint la Grece qu 'à demi, quand on n'en peint que les grands hommes; ajoutons quelques traits à cette Peinture, \& disons aujourd'hui quelque chose des Dames. Si celles dont je ferai THistoire sont dignes d'entrer dans mes Annales, on leur devoit la justice que je leur rends...". Cf. anche la polemica sulla condizione di dipendenza delle donne dai loro padri o mariti e la constatazione che se la donna abbandona il proprio consorte è ritenuta "indegna di vivere".

Sulle ragioni che permisero questa enorme fioritura di opere delle donne sulle donne si veda G. CONTI ODORISIO, 1979, p. 48.

E da notare che il richiamo al modello plutarcheo è fortissimo e chiaramente espresso nel proemio dell'opera di Fanfani: “...// presente mio libro potrebbe parere un di più; $e$ qualcuno dirà per avventura che l'opera mia è un rifare il fatto. Chi per altro non voglia essere un po' maligno, e voglia guardar sottilmente la cosa per il suo verso, vedrà che esso è nuovo così per la forma come per l'intenzione. Scrivendo le presenti Vite, io ho avuto il proposito, come tutti gli altri, di ammaestrar le fanciulle con l'esempio...". 
si presenta largamente ispirato all'etica cattolica e finalizzato a dimostrare che come la natura ha creato la donna fisicamente diversa dall'uomo, destinandola a partorire e ad allattare, così è inevitabile che il compito a lei attribuito "nell'umano consorzio" sia diverso da quello affidato all'uomo. Questi è tenuto a provvedere al mantenimento della famiglia, a difendere lo stato anche con l'uso delle armi in caso di pericolo o di necessità estrema, ad attendere alla vita politica, che richiede esclusivamente all'uomo di prendere la parola nelle assemblee; la donna, al contrario, deve occuparsi unicamente della famiglia e dell'educazione dei figli ${ }^{56}$. Il significato di quest'opera, che propone cinquanta biografie esemplari in forma dialogica, come pubblica lettura di componimenti di volta in volta corretti dagli insegnanti, è già stato messo in luce da I. Porciani; secondo la studiosa, la lezione che scaturisce dal testo di Fanfani è tutta impostata sul ruolo domestico e materno della donna, che non era di certo chiamata ad imitare le grandi figure del passato, né le scienziate e le letterate che troppo poco spazio potevano dedicare nella loro vita alla maternità e alla carità ${ }^{57}$.

In una direzione opposta sembra andare invece il Plutarco femminile di F. Berlan, che abbandonando il metodo delle compilazioni e delle riduzioni, scelse di comporre un'opera che istruisse e dilettasse al tempo stesso $^{58}$. Nel proemio l'autore dice di voler raccogliere e tratteggiare in circa quindici capitoli quanto di più bello, di più caro, di più santo e di più sublime hanno offerto le fanciulle italiane nei tempi antichi e moderni. Ciascun capitolo è paragonato ad mazzo di fiori, che raccoglie, secondo il principio della varietà, molteplici esempi di virtù femminili. Berlan, uomo di scuola che rigettava l'opinione di quanti erano convinti che le donne non avessero la capacità necessaria per svolgere le professioni forensis ${ }^{59}$, dedica in quest'opera particolare attenzione non solo a Pellegrina Amoretti, ma anche ad Eleonora Fonseca Pimentel, l'eroina vittima della dominazione borbonica del 1799 elogiata per avere amato la propria patria e avere lottato coraggiosamente per essa, ma in particolare per avere denunciato - in qualità di scrittrice - il male compiuto dai dominatori ${ }^{60}$.

Dunque, non c'è dubbio che il $D e$ mulierum virtutibus di Plutarco si sia

56 P. FANFANI, Il Plutarco femminile. Libro di lettura e di premio approvato dal consiglio scolastico di Firenze, Milano, 1893, pp. 5, 8.

57 I. PORCIANI, 1991, pp. 310-311.

58 F. BERLAN, $1878^{2}$, nella prefazione.

59 Cf. le osservazioni di M. RAICICH, 1991, p. 176 nota 16.

60 F. BERLAN, $1878^{2}$, pp. 452-453. 
prestato in età moderna ad usi, letture ed interpretazioni antitetiche. Ciò si può riscontrare anche nei casi in cui il recupero dell'opuscolo è stato solo parziale ed ha riguardato qualche passo o personaggio dell'opera. Un richiamo al Гovalкív aperai si può riscontrare negli Emblemata di Andrea Alciato (1531), il quale può ritenersi il fondatore di un nuovo genere artistico-letterario del Rinascimento che cercava di ammaestrare coniugando parola ed immagine. L'importanza e il successo riscossi dall'opera di Alciato nella prima metà del Cinquecento sono da attribuirsi - come sostiene A. Pérez Jiménez - allo spirito moraleggiante e didascalico dell'epoca, che trovava negli scritti morali di Plutarco una preziosa fonte cui attingere $^{61}$. Il titolo deH'emblema 106: "mulieris famam non formam vulgatum esse oportere", sembra essere a tutti gli effetti la traduzione latina di Mul. Viri.,

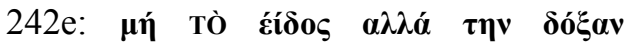

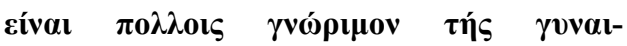
кós. Al centro del riquadro dell'emblema in questione campeggia l'immagine di Venere che tiene il piede destro sopra una tartaruga, simbolo del silenzio che la donna deve rispettare ${ }^{62}$. Che sia questo il messaggio implicito nella raffigurazione è confermato in modo inequivocabile dai versi sottostanti ad essa , che traducono in latino il passo $142 \mathrm{~d}$ dei Praecepta coniugalia.

In altri termini, Andrea Alciato ha reinterpretato in modo conservativo il passo del proemio del De mulierum virtutibus in cui Plutarco criticava il pregiudizio di Tucidide contro le donne ed ha eliminato, a tale scopo, ogni riferimento alla polemica sollevata dal Cheronese nei confronti della tradizione misogina precedente; ha poi ulteriormente rafforzato il suo punto di vista citando uno dei passi dei Praecepta coniugalia in cui Plutarco sostiene la necessità, per la donna, di rispettare nel matrimonio un ruolo tradizionale, cioè restare a casa e tacere .

Viceversa - come ha già sostenuto $\mathrm{G}$. Pomata $^{65}$ - nel 1583 Catherine des Roches si è richiamata nel Dialogue

61 A. Pérez JiMÉnez, 2003, pp. 375-402.

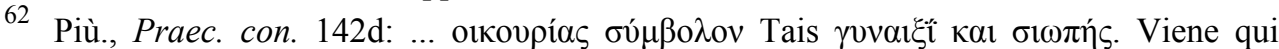
ripreso un concetto ampiamente diffuso nei tragici greci: cf. Aesch., Sept. 232 ed Eur.,

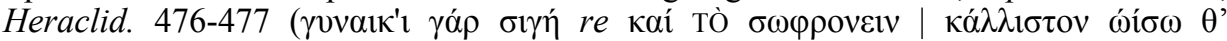

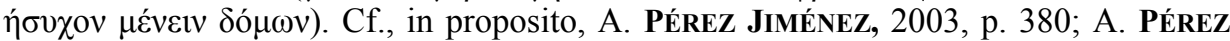
JIMÉNEZ, 2005, p. 803 e M. E. WIESNER, 2003, p. 17.1 simboli cui si ricorreva più spesso per rappresentare la moglie ideale erano la chiocciola e la tartaruga, animali che non abbandonano mai la casa e restano sempre in silenzio.

PLOUTARCHOS, n.s5 (2007/2008) 65-80. 
d'iris et Pasithée ad una delle protagoniste del De mulierum virtutibus, ovvero alla poetessa Telesilla (la donna d'Argo guarita da una malattia facendo voto di dedicarsi alle Muse) per sostenere il diritto della donna di rinunciare al matrimonio per dedicarsi allo studio delle lettere. Ancora, Telesilla e le Argive saranno menzionate nel secolo successivo da Lucrezia Marinelli tra gli esempi di quelle donne forti e intrepide, "che disprezzando la propria vita, hanno operate cose grandi e meravigliose con non poca invidia degli uomini" 66 .

E quindi evidente che la grande fortuna che le Virtù delle Donne di Plutarco hanno conosciuto nella modernità si deve agli utilizzi più disparati che si potevano riservare a singoli passi o personaggi dell'opera in differenti contesti e periodi storici. Camma è una delle figure femminili che hanno attirato l'attenzione non solo degli autori misogini, che videro in essa il modello esemplare della fedeltà coniugale, ma anche dei drammaturghi del Cinquecento e del
Seicento $^{67}$. Se la storia della regina dei Galati si prestava particolarmente bene ad un uso teatrale, perché certo non mancava di drammaticità, essendo molto

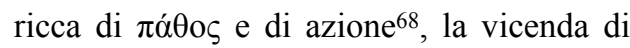
Aretafila viene letta in chiave politica nella prima metà del Seicento da P. Du Ryer, che scrive una tragicommedia dal titolo Arethaphile per compiacere i nobili francesi in rivolta contro Luigi XIII ${ }^{69}$.

\section{$\S$ 3. L'edizione delle Virtù delle} donne di Plutarco pubblicata a Piacenza nel 1794

Nel quadro delle edizioni moderne dell'opuscolo plutarcheo una segnalazione particolare merita quella piacentina alla quale si accennerà qui solo brevemente $^{70}$ : essa è una significativa testimonianza della fortuna conosciuta dal De mulierum virtutibus di Plutarco nel Settecento. Le Virtù delle donne di Plutarco vengono tradotte da tre letterati piacentini - Carlo Carasi, Giuseppe Gervasi e Federigo Scotti della Scala in occasione del matrimonio del nobile

66 Cf. il commento di G. ConTI OdoRISIO, 1979, pp. 126, 137.

67 Come fa notare J. Boulogne, Plutarque. Ouvres Morales, tome IV, Paris, 2002, p. 39, la vicenda della Galata Camma dà il nome ad un'opera di Montreux del XVI secolo; una Cammate è stata composta anche da J. de Hays; nel XVII secolo T. Corneille è autore di una Camma, reine de Galatie.

F. C. BABBITT, Plutarch's Moralia in fifteen volumes, III, London, 1968, pp. 550-551.

P. Du RYER, Arétaphile tragi-comédie. Texte établi et présenté par R. G. Zardini Lana, Genève-Paris, 1983, p. 19: "Arétaphile «était bien faite pour plaire à une aristocratie en révolte larvée contre le pouvoir royal»".

70 Per una trattazione più estesa si rimanda ad un altro articolo, di prossima pubblicazione sul Bollettino Storico Piacentino, incentrato sull'analisi e l'inquadramento storico di tale edizione. 
Luigi del Verme con la contessa Lucrezia Anguissola. L'opera costituisce quindi un nuptialium, ovvero un dono di nozze che venne edito a Piacenza nel 1794 da Giuseppe Tedeschi, tipografo, libraio e scrittore.

L'edizione piacentina delle Virtù delle donne non è, però, una traduzione integrale dell'opera composta in origine da Plutarco: essa contiene, infatti, solo dodici esempi di azioni virtuose rispetto ai ventisette narrati in principio dal Cheronese. I traduttori hanno quindi ridotto drasticamente il numero degli exempla di virtù femminile da includere nel dono di nozze, mostrando di preferire nettamente gli episodi dei quali erano state protagoniste donne che si potevano considerare massima espressione di pietas, accondiscendenza e fedeltà coniugale ${ }^{71}$.

Ritengo, tuttavia, che non siano solo ragioni di pura circostanza, di genere o d'occasione a giustificare la selezione operata dai tre traduttori. Nel 1794 la città di Piacenza era infatti sottomessa al dominio tirannico dei Borbone che avevano cercato in tutti i modi possibili di ostacolare ogni forma di "emancipazione" femminile, finendo oltretutto per soffocare nel sangue i tentativi di ribellione al regime monarchico sorti da iniziativa femminile: basti qui ricordare il caso più eclatante di Eleonora Fonseca Pimentel, condannata a morte nel 1799 per avere osato denunciare sul Monitore Napoletano le malefatte di Ferdinando IV di Borbone.

Il nuptialium in questione è pertanto anche un documento storico, uno specchio dei tempi in cui è stato prodotto. È degno di nota che i traduttori abbiano eliminato tutti quegli episodi nei quali la donna - vestendo i panni della guerriera (si pensi alle Celtiche) o della letterata (si pensi a Telesilla) - assume ruoli considerati dalla società del tempo tipicamente maschili e, pertanto, assolutamente sconvenienti.

\section{BiBLIOGRAFIA}

Aulotte, R.,

- "Plutarque et l'humanisme en France et en Italie", in M. IsHIGAMI-IAGOLNITZER (ed.), Les Humanistes et VAntiquité Grecque, Paris, 1989, pp. 99-104.

BERLAN, F.,

- Le fanciulle celebri e Tinfanzia delle donne illustri d'Italia antiche e moderne, Milano, $1878^{2}$.

Boulogne, J.,

- Plutarque et Teducation des femmes, in M. JuFRESA, F. Mestre, P. Gômez \& P. GILABERT (eds.), Plutarc a la seva època: paideia i societal Actas del Vili Simposio Espahol sobre Plutarco (Barcelona, 6-8 de Novembre de 2003), Barcelona, 2005, pp. 225-233.

BLOMQVIST, K.,

- "From Olympias to Aretaphila. Women in Politics in Plutarch", in J. MossmaN

${ }^{71}$ Non sembra quindi casuale che nel dono di nozze siano state inserite le vicende relative alle Focesi, alle Chiote, alle donne di Melo, alle Tirrene, alle Salmatidi, alle Milesie, a Pieria, Policrita, Camma, Chiomara, Timoclea, alla moglie di Piteo. 


\section{Tradizione, novità e fortuna in età moderna del De mulierum virtutibus di Plutarco 79}

(ed.), Plutarch and his Intellectual World, London, 1997, pp. 73-97.

CONTI ODORISIO, G,

- Donna e società nel Seicento. Lucrezia Marinelli e Arcangela Tarabotti, Roma, 1979.

GERA, D.,

Warrior Women. The Anonymous

Tractatus De Mulieribus, Leiden, New York, Kõln, 1997.

GIARELLI, F.,

- Storia di Piacenza. Dalle origini ai nostri giorni, vol. II, Piacenza, 1889.

GuZMÁn Guerra, A.

- "De virtutibus mulierum". Versis in graecum ex texto latino a C. Lascari, in A. PÉREZ JimÉnEZ \& G. DEL CERro CALDERÓN (eds.), Estudios sobre Plutarco: obra y tradición. Actas del I Symposion español sobre Plutarco. Fuengirola 1988, Malaga, 1990, pp. 265-270.

HIRZEL, R.,

- Plutarch, Leipzig, 1912.

O'BRIEN WICKER, K.,

"Mulierum virtutes (Moralia 242E-263C)", in H. Dieter Betz (ed.), Plutarch s Ethical Writings and Early Christian Literature, Leiden, 1978, pp. 106-134.

PÉREZ JIMÉNEZ, A.,

- "Los héroes de Plutarco como modelo en la literatura emblemática europea de los siglos XVI-XVII", in A. BARZANÓ, C. BEARZOT, F. LANDUCCI, L. PRANDI, G ZECCHINI (eds.), Modelli eroici dall-antichità alla cultura europea Bergamo, 20-22 novembre 2001,
Roma, 2003, pp. 375-402.

"Usos didácticos de la imagen y la palabra. El Plutarco de Juan Francisco de Villava", in M. JufresA, F. Mestre, P. GÓMEZ \& P. GILABERT, Plutarc a la seva època: paideia $i$ societat. Actas del Vili Simposio Español sobre Plutarco (Barcelona, 6-8 de Novembre de 2003), Barcelona, 2005, pp. 797-808.

Pomata, G,

"Storia particolare e storia universale: in margine ad alcuni manuali di storia delle donne", Quaderni storici, 74 (1990) 341385 .

PORCIANI, I.,

"Il Plutarco femminile", in S. SOLDANI (ed.), L'educazione delle donne. Scuole e modelli di vita femminile nell Italia dell Ottocento, Milano, 1991, pp. 297-317.

RAICICH, M.

"Liceo, università, professioni: un percorso difficile", in S. SOLDANI (ed.), $L$ 'educazione delle donne. Scuole e modelli di vita femminile nell'Italia dell'Ottocento, Milano, 1991, pp. 147 181.

RUSSELL, D. A.,

- Plutarch, London, 1973.

WIESNER, M. E.,

- Le donne nell'Europa moderna 15001750, trad, di DANIELA ARAGNO, Torino, 2003.

ZIEGLER, K.

Plutarco, ed. italiana a cura di B. ZUCCHELLI, traduzione di M. R. ZANCAN RINALDINII, Brescia, 1965. 